\title{
Secondary Frost Heave in Freezing Soils
}

\author{
A. C. Fowler
}

SIAM Journal on Applied Mathematics, Vol. 49, No. 4. (Aug., 1989), pp. 991-1008.

Stable URL:

http://links.jstor.org/sici?sici=0036-1399\%28198908\%2949\%3A4\%3C991\%3ASFHIFS\%3E2.0.CO\%3B2-I

SIAM Journal on Applied Mathematics is currently published by Society for Industrial and Applied Mathematics.

Your use of the JSTOR archive indicates your acceptance of JSTOR's Terms and Conditions of Use, available at

http://www.jstor.org/about/terms.html. JSTOR's Terms and Conditions of Use provides, in part, that unless you have obtained prior permission, you may not download an entire issue of a journal or multiple copies of articles, and you may use content in the JSTOR archive only for your personal, non-commercial use.

Please contact the publisher regarding any further use of this work. Publisher contact information may be obtained at http://www.jstor.org/journals/siam.html.

Each copy of any part of a JSTOR transmission must contain the same copyright notice that appears on the screen or printed page of such transmission.

The JSTOR Archive is a trusted digital repository providing for long-term preservation and access to leading academic journals and scholarly literature from around the world. The Archive is supported by libraries, scholarly societies, publishers, and foundations. It is an initiative of JSTOR, a not-for-profit organization with a mission to help the scholarly community take advantage of advances in technology. For more information regarding JSTOR, please contact support@jstor.org. 


\title{
SECONDARY FROST HEAVE IN FREEZING SOILS*
}

\author{
A. C. FOWLER $\dagger$
}

Abstract. A mathematical model of secondary frost heave in freezing, gravelly soils is presented. This model is due to O'Neill and Miller [Water. Resour. Res., 21 (1985), pp. 281-296], who sought numerical solutions. Here, their model is made nondimensional and is analysed using asymptotic analysis. Based on the successive formation of ice lenses within the partially frozen fringe, a heave criterion for such soils is deduced.

Key words. frost heave, reactive two-phase media, frozen soil, mushy zone, ice lensing

AMS(MOS) subject classifications. 76S05, 76T05, 35C20, 86A99

\section{NOMENCLATURE}

$c_{p} \quad$ specific heat

$f(W) \quad$ soil-ice-water characteristic curve

g gravity

I ice volume fraction

$K \quad$ dimensionless hydraulic conductivity

$k_{0} \quad$ hydraulic conductivity scale

$k_{h} \quad$ hydraulic conductivity

$k$ thermal conductivity

$L \quad$ latent heat

$n \quad$ pore volume fraction

$P \quad$ total pressure

$p \quad$ pore pressure; also used for the dimensionless water pressure, from (2.26) onward

$p_{e} \quad$ effective pressure

$p_{w} \quad$ water pressure

$p_{i} \quad$ ice pressure

$p_{b} \quad$ basal pressure

$S \quad$ rate of freezing

$t$ time

$T$ temperature

$T_{s} \quad$ surface temperature

$T_{b} \quad$ basal temperature

$U$ water flux

$V \quad$ ice flux

$v_{i} \quad$ ice (regelation) velocity

$W \quad$ water volume fraction

$z \quad$ vertical coordinate

$z_{s} \quad$ surface position

$z_{l} \quad$ base of (lowest) lens position

$z_{f} \quad$ base of frozen fringe position

$z_{b} \quad$ base of sample

* Received by the editors November 30, 1987; accepted for publication (in revised form) August 25, 1988.

$\dagger$ Mathematical Institute, Oxford University, 24-29 St. Giles, Oxford OX1 3LB, United Kingdom. This research was partially supported under grant N00017-86-G-0021 of the United States Navy to Doctor J. R. Ockendon at Oxford University, Oxford, United Kingdom. 


$\begin{array}{ll}\beta & \text { dimensionless parameter } \\ \gamma & \text { permeability exponent } \\ \delta_{1}-\delta_{4} & \text { dimensionless parameters } \\ \kappa & \text { thermal diffusivity } \\ \Pi & \text { osmotic pressure } \\ \rho_{w} & \text { water density } \\ \rho_{i} & \text { ice density } \\ \rho & \text { average density } \\ \rho_{s} & \text { solid density } \\ \theta_{s} & \text { dimensionless surface temperature } \\ \theta_{b} & \text { dimensionless basal temperature } \\ \chi & \text { weighting factor for pore pressures }\end{array}$

1. Introduction. Secondary frost heave (O'Neill [6]) is that phenomenon that occurs in freezing soils when the surface is forced upwards by the accumulation of horizontal ice "lenses" in the soil; see Fig. 1. The mechanism by which the ground is forced upwards is that water is drawn from below to the freezing front where it freezes. It is the upward motion of excess water that causes the heave, rather than simply the expansion of water on freezing. The water flow upwards is driven by a pressure gradient (or free energy gradient) that derives from the well-known fact (Hillel [3]) that in a moist soil, the water "pressure" is below the overburden pressure by an amount (the effective pressure) that depends on water content. The physical cause of this is a suction due both to capillary forces and to adsorption forces that cause an electrical double layer of water to form at the surfaces of clay particles. Thus the water pressure $p_{w}$ is lower where the water content is lower (i.e., where freezing occurs), and so the water flows upwards. The problem is to quantify and predict the amount and rate of heaving that can occur.

Soil is a complex medium which has complicated physical properties. Soils vary in texture from coarse sands and gravels to finer silts and the finest clays. Clay particles have typical dimension less than $2 \mu \mathrm{m}$. Compressibility, permeability, and effective pressure can vary enormously depending on the texture, and so do the heaving properties. Our approach will therefore be to adopt the simplest model we can. In addition, soil is a multiphase medium. Dry soil is soil and air; saturated soil is soil and water; partially frozen unsaturated soil is then soil, water, ice, air; and if we included the important effects of solutes, we should have four phases with five components-or six, if ice and water solute concentrations are both included.

There are two modes of frost heave. Primary frost heave occurs when all freezing takes place at an interface between pure ice and wet soil below. The water flows upwards through the soil and freezes due to a discontinuity in heat flux at the interface, just as in a Stefan problem. This phenomenon has been investigated by Jackson, Uhlmann, and Chalmers [4], for example. Secondary frost heave is of more interest (it heaves larger loads), and in this case the water freezes over a finite region below the ice lens, termed the partially frozen fringe. It thus resembles (in this) the mushy zone in a dendritically freezing alloy, and in a similar way the freezing temperature varies through the frozen fringe: in an alloy it is because the freezing (liquidus) temperature depends on solute concentration. This may also be important in soils, but additionally the freezing temperature varies with water pressure, which provides a similar effect.

The concept of effective pressure in soils is quite subtle, and even more so in partially frozen (or unsaturated) soils. Basically, a soil is a connected granular medium 


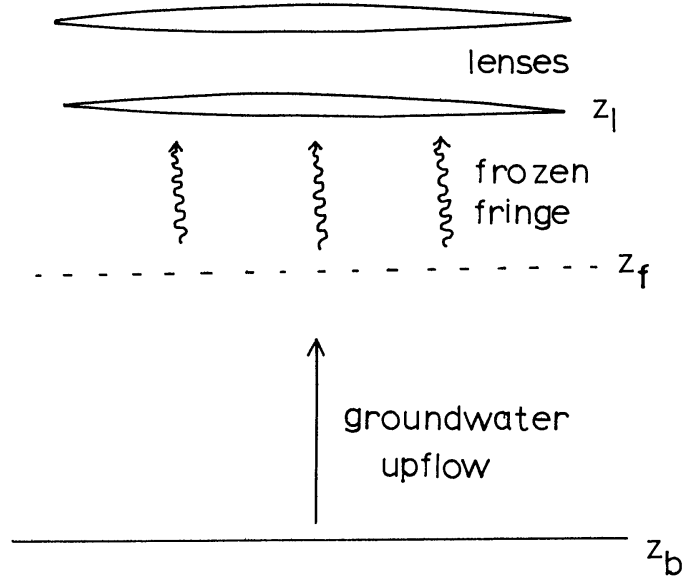

FIG. 1. Secondary frost heave. Ice lenses form in the soil due to a water flux upwards, driven by a capillary pressure gradient.

whose pores contain water (and/or air and ice), and this granular skeleton will in general support only part of an externally applied stress (e.g., overburden pressure). This pressure is the effective pressure $p_{e}$. The remainder must be supported by the pore fluids and thus

$$
P=p+p_{e},
$$

where $p$ is pore pressure, $P$ is overburden pressure. O'Neill [6] extends the concept of effective pressure to the case where there are two pore constituents, either water and air (saturated water vapour) or water and ice. The case of coexisting water, air and ice is discussed by Miller [5], who shows that it tends to be mechanically unstable, so that in that case, we might expect a sudden transition in pore constituents from water/air to water/ice, although this transition also might occur over a finite range of depth. The generalisation of (1.1) offered by O'Neill and Miller [7] is then

$$
p=\chi p_{w}+(1-\chi) p_{i},
$$

where $p_{w}$ and $p_{i}$ are water and ice pressures, and $\chi$ is a weighting factor, which in a simple approach we would take as

$$
\chi=W / n,
$$

where $W$ is the water volume fraction, and $n$ is the porosity (pore volume fraction). O'Neill and Miller [7] suggest that theory and experiments are consistent with the assumption

$$
p_{i}-p_{w}=f(W)
$$

where $f>0$ represents capillary effects between ice and water.

Since the equations we shall propose are appropriate to those for a two (or multi-) phase medium, it is important to be clear on the nature of the pressures described. In particular, two-phase flow theory commonly refers to phase-averaged pressures, which 
are the local averaged pressures, taken over the relevant phase (Drew [1]). In contrast, we have here defined total pressures, where the local average is taken over the whole control volume. If we denote the phase-averaged pressures by an overtilde, then we have, for example, that the total pore pressure $p$ is related to the phase-averaged pore pressure $\tilde{p}$ by $p=n \tilde{p}$. The particular pressures $p_{w}$ and $p_{i}$ introduced by O'Neill and Miller [7] to satisfy (1.2) are then related to the phase-averaged values, which we take to satisfy $\tilde{p}=\chi \tilde{p}_{w}+(1-\chi) \tilde{p}_{i}$, by $p_{w}=n \tilde{p}_{w}, p_{i}=n \tilde{p}_{i}$.

The point is that we might expect the characteristic relation (1.4) to apply to the phase-averaged pressures, since it refers essentially to a local interfacial property. For example, the effect of capillarity could be represented by $\tilde{p}_{i}-\tilde{p}_{w}=\sigma_{i w} \bar{\kappa}$, where $\sigma_{i w}$ is surface tension, and $\bar{\kappa}$ is the local averaged ice-water interface curvature. Then $p_{i}-p_{w}=$ $n \sigma_{i w} \bar{\kappa}(W)$, which is consistent with (1.4). In any case, the function $f(W)$ is inferred from the soil-moisture characteristic curve, which is experimentally measured (see O'Neill [6]). In particular, $f^{\prime}<0, f \rightarrow \infty$ as $W \rightarrow 0, f(n)=0$ : see Fig. 2.

\section{$f(W)$}

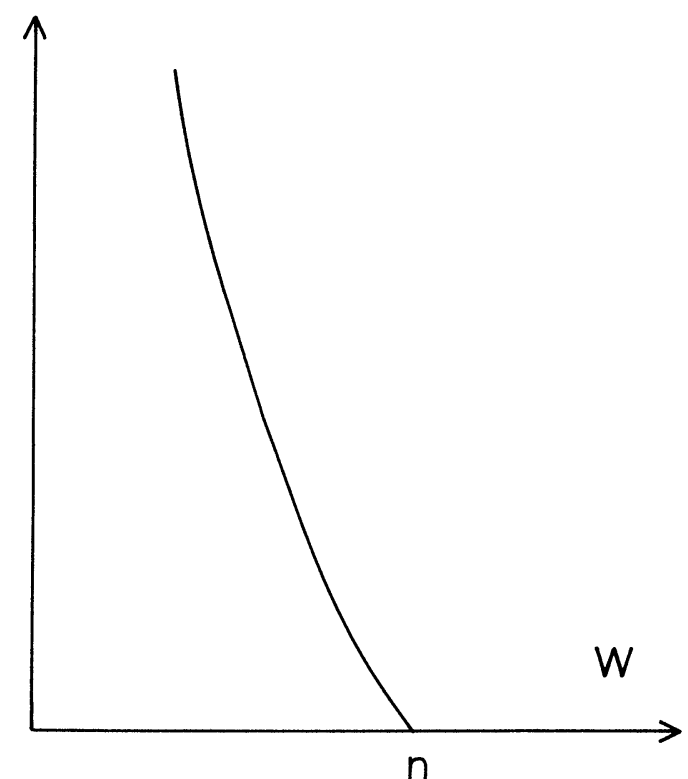

FIG. 2. Typical form for the frozen soil moisture characteristic curve.

There is an extensive literature on frost heaving and models thereof, which has been reviewed by O'Neill [6], and we do not wish to repeat such a survey. In the next section we recall the model of O'Neill and Miller [7], and only add here that it seems to be the most complete model for secondary frost heave yet to be presented. Although the model proposed consists of 10 equations (two mass, two momentum, one energy, plus five constitutive relations) for 10 unknowns, we shall find that a great degree of simplification ensues. The basic variables are the water pressure $p_{w}$, and the water fraction $W$. These are related through Darcy's law, which gives a first-order differential equation for $p_{w}$. The energy equation essentially gives a convective diffusion equation for $W$, since internal energy is essentially proportional (via latent heat) to $W$, while heat conduction is related to $W$ through temperature via $p_{w}$ (using the thermodynamic 
Clapeyron freezing curve and the soil-moisture characteristic curve). Despite this simplification, the boundary conditions are relatively complicated: there are two free boundaries, and one unknown coefficient in the equation for $W$, so that five boundary conditions are attached to it and these couple $p_{w}$ with $W$.

2. Mathematical model. We consider a situation such as that shown in Fig. 1. We suppose that the heat and mass transport is essentially one-dimensional, with $z$ the vertical coordinate. Let $z_{s}$ denote the ground surface, let $z_{l}$ be the base of the (lowest) ice lens, and also the top of the frozen fringe, let $z_{f}$ be the base of the frozen fringe, and let $z_{b}$ be the base of the sample under consideration. In the laboratory $z_{b}$ is finite; in the field $z_{b}$ might be at the level of the water table, or might be appropriately taken as $-\infty$. In the frozen fringe, O'Neill and Miller's [7] model can be written in the following form:

$$
\begin{aligned}
& W_{t}+U_{z}=-S / \rho_{w}, \\
& I_{t}+V_{z}=S / \rho_{i} ;
\end{aligned}
$$

these represent conservation of mass for water and ice phases. $U$ and $V$ represent water and ice fluxes, $W$ and $I$ are water and ice volume fractions, and $S$ is the rate of freezing (which will be determined from the energy equation). The water flux is determined by Darcy's law:

$$
U=-\frac{k_{h}}{\rho_{w} g}\left[\frac{\partial p_{w}}{\partial z}+\rho_{w} g\right]
$$

where $k_{h}$ is the hydraulic conductivity. The ice is considered to form a rigid, solid body, which is nevertheless able to flow relative to the soil skeleton by thermally induced regelation (see O'Neill and Miller [7]). The velocity of this flow will be determined from the boundary conditions. Thus we put

$$
V=I v_{i}
$$

where $v_{i}(t)$ is a function of time only. The energy equation can be written in the form

$$
-L S+\rho c_{p} \frac{d T}{d t}=k T_{z z},
$$

where $L$ is latent heat, $c$ is specific heat, and $k$ is thermal conductivity. We have assumed $k$ and $c$ are equal for the two phases, otherwise averaged values must be used. The density is also a weighted average, which we shall however take as constant.

In the frozen fringe, we assume thermodynamic equilibrium. This implies

$$
L\left(T-T_{0}\right) / T_{0}=\left(p_{w}-\Pi\right) / \rho_{w}-p_{i} / \rho_{i} ;
$$

see O'Neill and Miller [7] for a discussion of this generalised Clapeyron equation. It includes both the Clapeyron effect and the Gibbs-Thomson effect, as well as the effect of solutes via the osmotic pressure $\Pi$. The capillary relation is

$$
p_{i}-p_{w}=f(W),
$$

and the total pressure balance is

$$
P=p_{e}+\chi p_{w}+(1-\chi) p_{i},
$$

where

$$
\chi=\chi(W), \quad \text { e.g., } \chi=W / n \text {. }
$$


With these pressures, we require two constitutive relations to fully determine the problem. One is (2.7); the other depends on the characteristics of the soil. For a gravelly soil, which is relatively incompressible, we assume

$$
I+W=n
$$

is constant. In future work, we shall seek to use a characteristic relation to relate $p_{e}$ to $n$, as applicable to clays, for example, since these are more susceptible to frost heave (as we shall see this is because they are less permeable). That will also raise the thorny issue of soil hysteresis during freezing and thawing.

Equations (2.1)-(2.8) and (2.10) are nine equations for the 10 unknowns $W, U$, $S, I, V, p_{w}, v_{i}, T, p_{i}, p_{e}$, providing $P$ and $\Pi$ are prescribed. We will hence forward neglect $\Pi$, i.e.,

$$
\Pi=0 \text {, }
$$

and assume that $P$ is determined from (quasi-) static equilibrium. The simplest assumption is that $P$ is hydrostatic, i.e.,

$$
P=P_{0}-\rho_{s} g\left(z-z_{s}\right),
$$

where $\rho_{s}$ is the soil density (considered constant here). We assume that the permeability $k_{h}$ is given by

$$
k_{h}=k_{0}(W / n)^{\gamma}
$$

(O’Neill and Miller [7]). Thus, we are missing one equation to determine $v_{i}$ : this will come from an extra boundary condition.

The model above, which applies in the frozen fringe, must be supplemented by the temperature equation in the frozen region above and equations of temperature and water flow below. We denote the boundaries of the frozen fringe by $z_{l}$ (at the top) and $z_{f}$ (at the bottom). As illustrated in Fig. 1, we assume that an ice lens forms the upper boundary $z_{l}$ (and may be one of several above the fringe). Therefore, above the fringe: in $z>z_{l}$,

$$
\frac{d T}{d t}=\kappa T_{z z},
$$

where $\kappa=k / \rho c_{p}$ is the thermal diffusivity. Also, below the fringe: in $z<z_{f}$,

$$
\begin{aligned}
& W_{t}+U_{z}=0, \quad U=-\frac{k_{h}}{\rho_{w} g}\left[\frac{\partial p_{w}}{\partial z}+\rho_{w} g\right], \\
& \frac{d T}{d t}=\kappa T_{z z}, \quad P=p_{e}+p_{w}, \quad W=n .
\end{aligned}
$$

The boundary conditions that we will seek to apply to these equations are the following:

at $z=z_{s}$ :

$$
T=T_{s}
$$

(the surface temperature is prescribed);

at $z=z_{l}$ :

$$
\begin{aligned}
& \rho_{i}\left(v_{i}-\dot{z}_{l}\right)(1-I)=\rho_{w}\left(U-W \dot{z}_{l}\right), \quad p_{i}=P, \\
& \rho_{w} L\left(U-W \dot{z}_{l}\right)=\left[-k \frac{\partial T}{\partial z}\right]_{-}^{+}, \quad[T]_{-}^{+}=0
\end{aligned}
$$


global conservation of mass:

$$
\left.\rho_{w} U\right|_{z_{b}}=\rho_{i} v_{i}
$$

at $z=z_{f}$ :

$$
\begin{aligned}
& W=n, \quad[T]_{-}^{+}=0, \\
& {\left[\frac{\partial T}{\partial z}\right]_{-}^{+}=0, \quad[p]_{-}^{+}=0, \quad[U]_{-}^{+}=0 ;}
\end{aligned}
$$

at $z=z_{b}$,

$$
T=T_{b}, \quad p=p_{b} \quad \text { (see below). }
$$

These conditions represent applied surface temperature (2.16), conservation of mass $(2.17)_{1}$, stress continuity $(2.17)_{2}$, energy conservation (Stefan condition) $(2.17)_{3}$, thermodynamic equilibrium $(2.17)_{4}$, conservation of mass (2.18), definition of fringe base $(2.19)_{1}$, thermodynamic equilibrium $(2.19)_{2}$, energy conservation $(2.19)_{3}$, stress continuity $(2.19)_{4}$, mass conservation $(2.19)_{5}$, applied basal temperature and pressure (2.20). They are straightforward, except $(2.19)_{1}$, which is an extra boundary condition necessary to close the problem. It is a common procedure in two-phase solidification problems that such an extra condition has to be postulated (cf. Worster [9]). The boundary condition (2.20) at the base of the domain merits some further discussion. In the laboratory, $z_{b}$ represents the actual base of the sample, and (2.20) would certainly be appropriate. The same is reasonable in the field, if $z_{b}$ represents the location of the water table, although this really requires the soil to be unsaturated. We might also be tempted to consider $z_{b} \rightarrow-\infty$ (as we shall in fact do in $\S 3$ ), but we must be careful about how this is done (see the further comments after (2.37)).

Procesding, we now nondimensionalise the equations. We do this by writing

$$
\begin{aligned}
& U=[U] U^{*}, \quad V=[U] V^{*}, \quad z=[z] z^{*}, \quad S=[S] S^{*}, \quad t=[t] t^{*}, \\
& p_{w}=P_{0} p_{w}^{*}, \quad p_{e}=P_{0} p_{e}^{*}, \quad p_{i}=P_{0} p_{i}^{*}, \quad T=T_{0}+[T] T^{*},
\end{aligned}
$$

where the scales in (2.21) are chosen as follows. We anticipate that latent heat is large, and thus from (2.5),

$$
L[S]=k[T] /[z]^{2} .
$$

We also choose a convective time scale

$$
[t]=[z] /[U],
$$

and balance $(2.1)$ by choosing

$$
[S]=\rho_{w}[U] /[z]
$$

Equation (2.6) suggests

$$
[T]=T_{0} P_{0} / \rho_{i} L,
$$

and we take the length scale $[z]$ as prescribed, e.g., $[z] \leqq 1 \mathrm{~m}$ for laboratory tests.

The equations can be written in dimensionless form, where we henceforth drop the asterisks (having written $f(W)=P_{0} f^{*}(w)$ ):

$$
\begin{aligned}
& \left(W_{t}+U_{z}\right)=-S, \quad\left(I_{t}+v_{i} I_{z}\right)=\frac{S}{1-\delta_{3}}, \\
& U=-\beta K\left[\frac{\partial p}{\partial z}+\delta_{1}\right], \quad T=-f(W)-\delta_{3} p, \quad-S+\delta_{2} \frac{d T}{d t}=T_{z z}, \\
& 1-\delta_{4}\left(z-z_{s}\right)=p_{e}+\chi p+(1-\chi) p_{i}, \quad p_{i}-p=f(W), \quad I+W=n,
\end{aligned}
$$


in the frozen fringe, where

$$
\begin{aligned}
& \delta_{1}=\rho_{w} g[z] / P_{0}, \quad \delta_{2}=[U][z] / \kappa, \quad \delta_{3}=1-\rho_{i} / \rho_{w}, \\
& \delta_{4}=\rho_{s} g[z] / P_{0}, \quad \beta=\rho_{i} k_{0} L^{2} / g T_{0} k,
\end{aligned}
$$

and we have written $p_{w}=p$. The dimensionless hydraulic conductivity is

$$
K=(W / n)^{\gamma} \text {. }
$$

In $z>z_{l}$,

$$
\delta_{2} \frac{d T}{d t}=T_{z z}
$$

while in $z<z_{f}$

$$
\begin{array}{ll}
W_{t}+U_{z}=0, \quad U=-\beta\left[\frac{\partial p}{\partial z}+\delta_{1}\right], & \delta_{2} \frac{d T}{d t}=T_{z z}, \\
1-\delta_{4}\left(z-z_{s}\right)=p_{e}+p, \quad W=n . &
\end{array}
$$

The boundary conditions are:

at $z=z_{s}$,

$$
T=-\theta_{s}
$$

at $z=z_{l}$,

$$
\begin{aligned}
& \left(1-\delta_{3}\right)\left(v_{i}-\dot{z}_{l}\right)(1-I)=U-W \dot{z}_{l}, \quad p_{i}=1-\delta_{4}\left(z_{l}-z_{s}\right), \\
& \left(U-W \dot{z}_{l}\right)=\left[-\frac{\partial T}{\partial z}\right]_{-}^{+}, \quad[T]_{-}^{+}=0
\end{aligned}
$$

global mass conservation

$$
\left.U\right|_{z_{b}}=\left(1-\delta_{3}\right) v_{i}
$$

at $z=z_{f}$,

$$
\begin{aligned}
& W=n, \quad[T]_{-}^{+}=0, \quad\left[\frac{\partial T}{\partial z}\right]_{-}^{+}=0, \\
& {[p]_{-}^{+}=0, \quad[U]_{-}^{+}=0,}
\end{aligned}
$$

on $z=z_{b}$,

$$
T=\theta_{b}, \quad p=p_{b},
$$

where

$$
\theta_{s}=\left(T_{0}-T_{s}\right) /[T], \quad \theta_{b}=\left(T_{b}-T_{0}\right) /[T] .
$$

To estimate the values of the parameters, we take $P_{0} \sim 1 \mathrm{bar}, \rho_{i} \sim 900 \mathrm{~kg} \mathrm{~m}^{-3}, \rho_{w} \sim$ $1000 \mathrm{~kg} \mathrm{~m}^{-3}, L \sim 3 \times 10^{5} \mathrm{~J} \mathrm{~kg}^{-1}, k \sim 2 \mathrm{~W} \mathrm{~m}^{-1} \mathrm{~K}^{-1}, c_{p} \sim 2 \mathrm{~kJ} \mathrm{~kg}^{-1} \mathrm{~K}^{-1}$. Values of $k_{0}$ can vary from $10^{-2} \mathrm{~cm} \mathrm{~s}^{-1}$ for sand to $10^{-8} \mathrm{~cm} \mathrm{~s}^{-1}$ for clay. We use $[z] \sim 10 \mathrm{~cm}$, a value used in some of their numerical tests by O'Neill and Miller [7]. We then find

$$
\begin{aligned}
& {[U] \sim 10^{-6} \mathrm{~cm} \mathrm{~s}^{-1}, \quad[T] \sim 0.1 \mathrm{~K}, \quad \delta_{1}, \delta_{4} \sim 10^{-2},} \\
& \delta_{3} \sim 10^{-1}, \quad \delta_{2}=\left(L / c_{p}[T]\right)^{-1} \sim 10^{-3}, \quad 10^{6} \geq \beta \geq 1 .
\end{aligned}
$$

The velocity scale here is representative of a significant rate of heave. The values of $\beta$ are large for gravelly soils and smaller for clays. We now simplify the model by seeking to adopt the approximations suggested by these estimates, namely that $\delta_{1}, \delta_{2}$, 
$\delta_{3}, \delta_{4}$ are all small. We retain $\beta$ for the moment, since it can be either large or moderate for different soils. Our previous remarks following (2.20) may now be amplified. If we try and apply (2.35) to (2.30) with $z_{b}=-\infty$, then if $\delta_{2} \ll 1$, we shall find $T \approx \theta_{b}$ on $z=z_{f}$, in apparent contradiction to $(2.26)_{4}$, which implies $T \approx 0$ there. In fact, the order of the limits $\delta_{2} \rightarrow 0, z_{b} \rightarrow-\infty$ is important. For this reason, we consider $z_{b}$ as finite, at least until $\delta_{2} \rightarrow 0$ has been considered. This is evidently appropriate, since in practice we expect $\left|z_{b}\right| \ll 1 / \delta_{2}$.

Let us neglect terms of order $\delta_{j}$ in the equations. Global conservation of mass (with $z_{b}=$ constant) is approximately

$$
\left.U\right|_{z_{b}}=v_{i}
$$

it further follows from (2.30) that $\left.U\right|_{z_{f}}=\left.U\right|_{z_{b}}$. Using (2.38) together with (2.26) $)_{1,2}$, we obtain

$$
U \approx v_{i}(1-n+W)
$$

in the frozen fringe. Thus equations for $W$ and $p$ in the fringe are, approximately,

$$
U=-\beta K \frac{\partial p}{\partial z}=v_{i}(1-n+W), \quad W_{t}+v_{i} W_{z}=-\frac{\partial^{2}}{\partial z^{2}} f(W) .
$$

We require two boundary conditions for $W$, one for $p$, together with two relations for $z_{l}$ and $z_{f}$ and one for $v_{i}$. The solution of (2.29) (with $\delta_{2}=0$ ), using (2.32), yields

$$
T \sim-\theta_{s}+G\left(z_{s}-z\right), \quad z>z_{l},
$$

where the surface $z_{s}$ is given by

$$
z_{s}=\int_{0}^{t} v_{i} d t
$$

(this follows from the assumption that the frozen soil in $z>z_{l}$ is incompressible: conservation of mass then implies that $(d / d t)\left(z_{s}-z_{l}\right)=v_{i}-\dot{z}_{l}$, whence (2.42) follows); $G$ follows from $(2.32)_{4}$ and $(2.26)_{4}$ :

$$
G=\left(\theta_{s}-f\right) /\left(z_{s}-z_{l}\right) .
$$

Substitution of (2.39) into (2.32) $)_{1}$ gives, approximately,

$$
\dot{z}_{l}=0, \quad z_{l}=\text { constant. }
$$

Conditions $(2.32)_{2}$ and $(2.32)_{3}$, using $(2.26)_{7},(2.44)$, and (2.43), give the following conditions at $z_{l}$ :

$$
U=-f_{z}+\frac{\theta_{s}-f}{\left\{\int_{0}^{t} v_{i} d t-z_{l}\right\}}, \quad p+f(W)=1 .
$$

Solution of (2.30) in $z<z_{f}$ (with $\delta_{2}=\delta_{4}=0$ ). gives linear profiles for $T$ and $p$ there; then the boundary conditions (2.34) give

$$
W=n, \quad f_{z}=\frac{\theta_{b}}{z_{f}-z_{b}}, \quad-\frac{\partial p}{\partial z}=\frac{p_{b}-p}{z_{f}-z_{b}},
$$

where we have assumed $f(n)=0$ (see Fig. 2).

Finally, we define a criterion for lens formation. Ice lenses can form if the effective pressure decreases to zero anywhere (because there is then no stress to keep soil grains in contact). From (2.26), the criterion for this may be written as

$$
p+(1-\chi) f(W)=1 \text {. }
$$


If this criterion is satisfied at some point in $\left(z_{f}, z_{l}\right)$, then a lens is considered to form there, and the value of $z_{l}$ is shifted to that place (in the computations of O'Neill and Miller).

3. Analysis. Our aim in this section is to analyse the model to the extent we can, and in particular to provide some explanation and interpretation for the results obtained by O'Neill and Miller [7]. In particular, we wish to focus on the following ideas:

(a) "... gravels are not susceptible to heave. Silts can produce spectacular heave, but only if the load ... is small ... clays never produce spectacular heave, but can ... heave very large loads indeed" [7].

(b) Fig. 4 of [7] shows a "typical" freezing sequence of a $10 \mathrm{~cm}$ soil column. A sequence of very thin ice lenses forms at intervals of $\sim 10^{4} \mathrm{~s}$ and at distances apart of $\sim 0.1 \mathrm{~cm}$, these values increasing as time increases.

(c) Fig. 3 of [7] shows a "typical" variation of $p$ and $f(W)$ (here: $u_{w}$ and $-T$ in their figure). The fringe thickness is $\sim 1 \mathrm{~cm}$, while $p$ jumps from its value at $z_{l}$ to the far field value ( $p_{b}$ : taken as zero in the figure) over a "boundary layer" of thickness $\sim 0.2 \mathrm{~cm}$. The profile for $f$, however, is almost linear.

(d) Fig. 5 of [7] shows cumulative heave profiles $h(t)$ of $O(2 \mathrm{~mm})$. There is an initial rapid rate of change of $h$ (over a time $\sim 10^{5} \mathrm{~s}$ ) followed by an almost linear increase of $h$ with $t$ for $t \leqslant 10^{6} \mathrm{~s}$. The rate of heave depends very sensitively on the applied load $P_{0}$, varying from about $3 \times 10^{-6} \mathrm{~cm} \mathrm{~s}^{-1}$ for $P_{0} \approx 0.9$ bar, to about $0.1 \times$ $10^{-6} \mathrm{~cm} \mathrm{~s}^{-1}$ for $P_{0} \approx 1.1$ bar.

For convenience we collect together the approximate model equations (2.40), (2.45), and (2.46) derived in the previous section. These are:

$$
-\beta K \frac{\partial p}{\partial z}=v_{i}(1-n+W), \quad W_{t}+v_{i} W_{z}=-\frac{\partial^{2}}{\partial z^{2}} f(W),
$$

in the frozen fringe $z_{l}>z>z_{f}$, with boundary conditions

$$
p+f(W)=1, \quad v_{i}(1-n+W)=-f_{z}+\frac{\theta_{s}-f}{H}
$$

on $z=z_{l}$ (constant), and

$$
W=n(f=0), \quad f_{z}=\frac{\theta_{b}}{z_{f}-z_{b}}, \quad p_{z}=\frac{p}{z_{f}-z_{b}},
$$

on $z=z_{f}$, where we have taken $p_{b}=0$ without loss of generality (so $P_{0}$ represents excess load). We assume $K=(W / n)^{\gamma}$, as in (2.28). The depth to the lowest lens is $H$, given by

$$
H=-z_{l}+h,
$$

where $h=z_{s}(t)-z_{s}(0)$ is the total heave, and

$$
\dot{h}=v_{i} \text {. }
$$

The relationship of $H, h$, and $z_{l}$ is illustrated in Fig. 3. After the onset of freezing, $z_{s}$ will increase with time. From (2.42), the rate of heaving $\dot{h}$ is simply $v_{i}$. Although $\dot{z}_{l}=0$ as heaving occurs, secondary lens formation causes $z_{l}$ to be relocated at the base of the new lens. In this way we will have $z_{l}<0$ in general. Thus the depth $H$ to the lowest lens, given by (3.4), will not in general be given by the total accumulated heave $h$. 


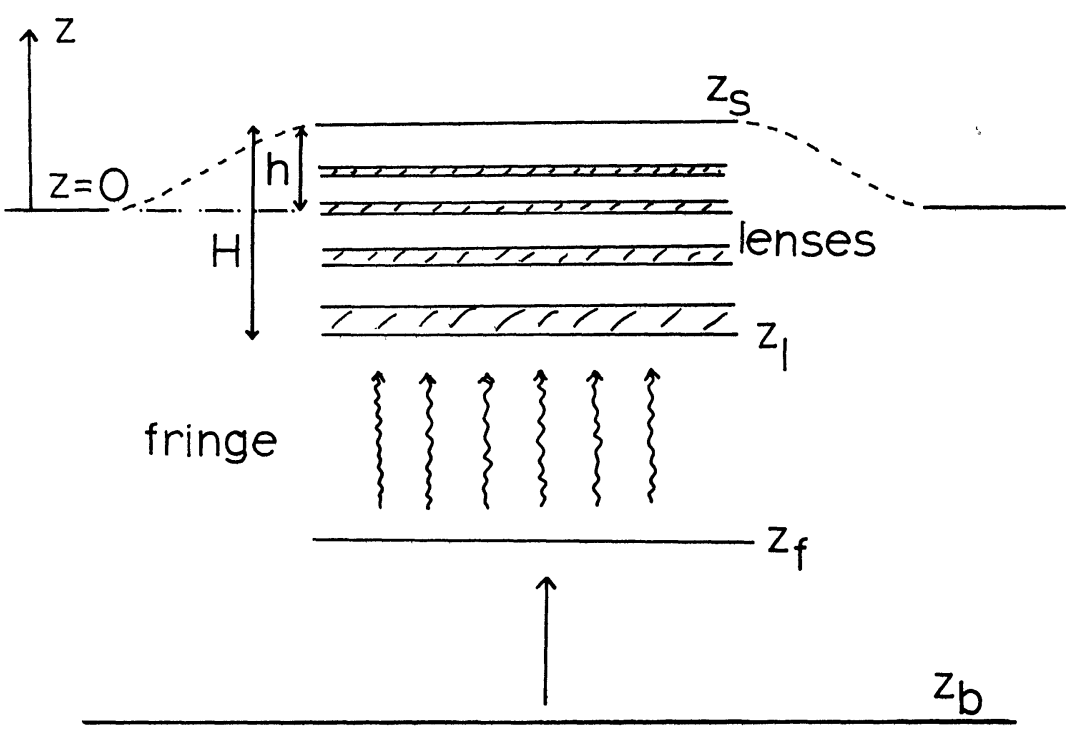

FIG. 3. Illustration of the relationship between $H$ and $h$.

We solve the above problem with $z_{l}=$ constant, and $h=h_{0}$ at $t=0$, until the lens formation criterion (2.47), i.e.,

$$
p+(1-\chi) f(W)=1
$$

is satisfied for some $z^{*}<z_{l}$, at $t=t^{*}$. At this point, following O'Neill and Miller [7] we would begin the calculation again, with new values of $z_{l}=z^{*}$ and $h_{0}=h_{0}+\int_{0}^{t^{*}} v_{i} d t$. The new initial profile for $W$ is just that at the end of the previous calculation $\left(t=t^{*}-\right)$. We can see that the third-order system (3.1) with two unknowns $v_{i}$ and $z_{f}$ has five boundary conditions, as appropriate. For the parameters considered by O'Neill and Miller [7], we have $\theta_{s} \sim \theta_{b} \sim 10$. They do not report values of $\beta$, but refer to measurements appropriate to a silty soil. Miller [5] reports measurements of Williams and Burt [8], which suggest that $k_{0} \sim 10^{-4} \mathrm{~cm} \mathrm{~s}^{-1}$, which would give $\beta \sim 10^{4}$. It is important to anticipate that the fringe permeability (which is rate-controlling) will be low at the lens, where $W=W_{l}$, say. Then $K=\left(W_{l} / n\right)^{\gamma}$ there, and if $W_{l} \leqq 0.2$ (consistent with $f>1$, see Fig. 2, or Fig. 2 of [7]), we have $K \sim 2 \times 10^{-3}$ for $\gamma=9$. Thus $\beta K_{l} \sim 1$ for $\beta \sim 10^{3}\left(k_{0} \sim 10^{-5} \mathrm{~cm} \mathrm{~s}^{-1}\right)$ and will be lower for smaller $W$. On this account, we do not yet consider any approximations based on the size of $\beta$.

We will restrict our attention to cases where similarity solutions are appropriate, that is, small time or, equivalently, letting $z_{f}$ and $z_{f}-z_{b}$ become infinite in (3.3). (See the comments after (2.37).) Initial conditions consistent with such a solution are those for step freezing, i.e., an initial sudden surface cooling. The boundary conditions (3.3) are replaced by

$$
W \rightarrow n(f \rightarrow 0), \quad p \rightarrow 0, \quad \text { as } z \rightarrow-\infty .
$$

(i) Large permeability exponent: $\gamma \gg 1$. The permeability exponent $\gamma$ has measured values of order seven or nine, according to O'Neill and Miller. This implies that the permeability $K$ changes rapidly, by several orders of magnitude, as $K$ increases below the lens. To take account of this, we use the idea of large activation energy asymptotics. Thus, for small $z_{l}-z$, we can write

$$
W \approx W_{l}-W_{l}^{\prime}\left(z_{l}-z\right),
$$


where $W_{l}=W\left(z_{l}, t\right), W_{l}^{\prime}=\partial W /\left.\partial z\right|_{z_{l}}$; hence

$$
K \approx\left(W_{l} / n\right)^{\gamma} \exp \left[\Lambda\left(z_{l}-z\right)\right]
$$

where

$$
\Lambda=\gamma\left|W_{l}^{\prime}\right| / W_{l}
$$

notice that $\Lambda$ will generally depend on $t$. Defining $B$ by

$$
B=\beta K_{l} \Lambda /\left(1-n+W_{l}\right),
$$

where

$$
K_{l}=\left(W_{l} / n\right)^{\gamma}
$$

we can rewrite (3.1) 1 approximately

$$
-\frac{\partial p}{\partial z} \approx\left(\frac{\Lambda}{B}\right) v_{i} \exp \left[-\Lambda\left(z_{l}-z\right)\right],
$$

when $z_{l}-z$ is small. Evidently, the solution of (3.13) gives a uniform approximation to $p$ (with $\Lambda \gg 1)$ :

$$
p \approx-\left(\frac{v_{i}}{B}\right) \exp \left[-\Lambda\left(z_{l}-z\right)\right]
$$

We denote

$$
f_{l}=f\left(W_{l}\right)
$$

then $(3.2)_{1}$ implies that the rate of heave is given by

$$
v_{i}=B\left(f_{l}-1\right),
$$

thus requiring $f_{l}>1$, and the problem is now reduced to solving

$$
W_{t}+v_{i} W_{z}=-f_{z z},
$$

with $v_{i}$ given by (3.16), and

$$
\begin{aligned}
& v_{i}(1-n+W)=-f_{z}+\left(\theta_{s}-f\right) / H \text { on } z_{l}, \\
& W \rightarrow n \quad(f \rightarrow 0) \quad \text { as } z \rightarrow-\infty .
\end{aligned}
$$

At this point we have not involved similarity, and more generally, (3.1) $)_{1,2}$ could be used instead of (3.19). Furthermore, no assumption on the magnitude of $B$ has been made. As a rough guide, we would associate $B \ll 1$ with clay, $B \sim 1$ with silt, and $B \gg 1$ with gravel. The two extreme limits $B \ll 1, B \gg 1$ yield the following. If $B \ll 1$ (clay), then $v_{i} \sim B, t \sim 1 / B, W$ is approximately linear, and the solution can be easily written down from the boundary conditions in (3.3). If $B \gg 1$, a self-consistent approximation has $v_{i} \sim O(1)$, and $f_{l} \approx 1$. In this case (3.14) implies that $|p| \ll 1$.

(ii) Similarity solution. A similarity solution to (3.17), with (3.18), (3.19), (3.16), (3.11), (3.10), (3.4), and (3.5) (with $z_{l}=0$ ) has

$$
W=g(\eta), \quad \eta=-z / 2 \sqrt{t}, \quad B=b / \sqrt{t}, \quad v_{i}=\alpha / \sqrt{t}, \quad H=2 \alpha \sqrt{t},
$$

and $g$ satisfies

$$
\frac{d^{2} f}{d \eta^{2}}=2(\alpha+\eta) \frac{d g}{d \eta}
$$


with

$$
g=g_{l} \quad \text { on } \eta=0, \quad g \rightarrow n \quad \text { as } \eta \rightarrow \infty
$$

and

$$
\begin{aligned}
& \alpha=b\left(f_{l}-1\right), \quad b=\left\{\frac{g_{l}^{\prime}}{2 g_{l}\left(1-n+g_{l}\right)}\right\}\left(\frac{g_{l}}{g^{*}}\right)^{\gamma}, \\
& \alpha\left(1-n+g_{l}\right)=\frac{1}{2} f_{l}^{\prime}+\frac{\theta_{s}-f_{l}}{\alpha},
\end{aligned}
$$

where we have defined

$$
g^{*}=n /(\beta \gamma)^{1 / \gamma}:
$$

we assume $g^{*}<n$. In (3.23), we have written $g_{l}, g_{l}^{\prime}, f_{l}, f_{l}^{\prime}$ for $g(0), g^{\prime}(0), f\left(g_{l}\right), d f /\left.d \eta\right|_{\eta=0}$.

To analyse this system, we again make use of the fact that $\gamma \gg 1$. Note that $g^{*}$ is not numerically much different from $n$. For $\beta \sim 1$, it is close to $n$, for large $\beta$, it may be smaller by a factor of two. There are three cases to consider:

(a) $g_{l}<g^{*}$. Then $b \ll 1, \alpha \ll 1$, and (3.23) is approximated by

$$
\theta_{s} \approx f_{l} \text {. }
$$

For $\alpha>0$, and to be self-consistent, we require $f_{l}>f^{*} \equiv f\left(g^{*}\right)$ (since $d f / d g<0$ ), thus

$$
\theta_{s}>f^{*}, \quad \theta_{s}>1 \text {. }
$$

(b) $g_{l} \approx g^{*}$. In this case $(3.23)_{2}$ gives the precise value of $g_{l},(3.23)_{1}$ gives $b$, and $\alpha$ is determined from $(3.23)_{3}$, which requires solutions of the differential equation. Self-consistency implies (since $f_{l}^{\prime}<0$ )

$$
\theta_{s}>f^{*}>1 \text {. }
$$

(c) $g_{l}>g^{*}$. This is similar to (b), except that $(3.23)_{2}$ implies $b \gg 1$, and hence $(3.23)_{1}$ gives

$$
f_{l} \approx 1, \quad g_{l} \approx g_{1}
$$

where $f\left(g_{1}\right)=1$, and $\alpha$ is again determined from the equation, but with $g=g_{1}$ at $\eta=0$. Validity of this case requires

$$
\theta_{s}>1 \text { and } f^{*}>1 \text {. }
$$

The regions of validity (3.26), (3.27), and (3.29) overlap in $1<f^{*}<\theta_{s}$, where all three solutions are possible. Thus the similarity solution we seek is not necessarily unique.

Explicit solution of (3.21) is not possible in general. Various possibilities occur: we can.write down the solution if $f(g)$ is linear; we can prove what we need about the variation of $f_{l}^{\prime}$ with $\alpha$, by just supposing $f(g)$ is monotonic. Equivalent results occur by approximating (3.21) by

$$
\frac{d^{2} f}{d \eta^{2}} \approx 2 \alpha \frac{d g}{d \eta}
$$

which is in fact a reasonable approximation for large loads or fine soils, and we choose this method as the results are most easily understood. The solution of (3.30), with the boundary conditions (3.22), is

$$
2 \alpha \eta=\int_{f}^{f_{t}} \frac{d f}{[n-g(f)]} .
$$


In particular,

$$
f_{l}^{\prime} \approx-2 \alpha\left(n-g_{l}\right)
$$

so that $(3.23)_{3}$ gives

$$
\alpha \approx\left(\theta_{s}-f_{l}\right)^{1 / 2} \text {. }
$$

We approximate $(3.23)_{2}$ and $(3.23)_{1}$ by

$$
\alpha \approx c\left(f_{l}-1\right)\left(f^{*} / f_{l}\right)^{\gamma},
$$

which for $c=O(1)$ adequately represents the behaviour of $\alpha$ as a function of $f_{l}$. Equating (3.33) and (3.34) enables us to eliminate $f_{l}$ and find $\alpha$ explicitly. The three cases (a), (b), (c) lead to the following:

$$
\begin{aligned}
& \text { (a) } g_{l}<g^{*}, \quad b \ll 1 ; \quad \theta_{s}<f^{*}, \quad \theta_{s}>1: \alpha \approx c\left(\theta_{s}-1\right)\left(f^{*} / \theta_{s}\right)^{\gamma} \text {; } \\
& \text { (b) } g_{l} \approx g^{*}, \quad b \sim 1 ; \quad \theta_{s}>f^{*}>1: \alpha \approx\left(\theta_{s}-f^{*}\right)^{1 / 2} \text {; } \\
& \text { (c) } g_{l}>g^{*}, \quad b \gg 1 ; \quad \theta_{s}>1, \quad f^{*}>1: \alpha \approx\left(\theta_{s}-1\right)^{1 / 2} \text {. }
\end{aligned}
$$

It is easy to trace the qualitative bifurcation structure by graphically portraying (3.33) and (3.34). It is less easy to show the $\alpha, \theta_{s}, f^{*}$ solution surfaces in three dimensions, however. In Fig. 4, we show slices of this surface, at fixed $f^{*}$ or $\theta_{s}$, and we attempt to draw the three-dimensional version in Fig. 5.

(iii) Lens formation. Successive lenses will form if the criterion (3.6) is satisfied anywhere. Now $p$ increases rapidly to zero near $z_{l}$, while beyond this $W$ increases, so
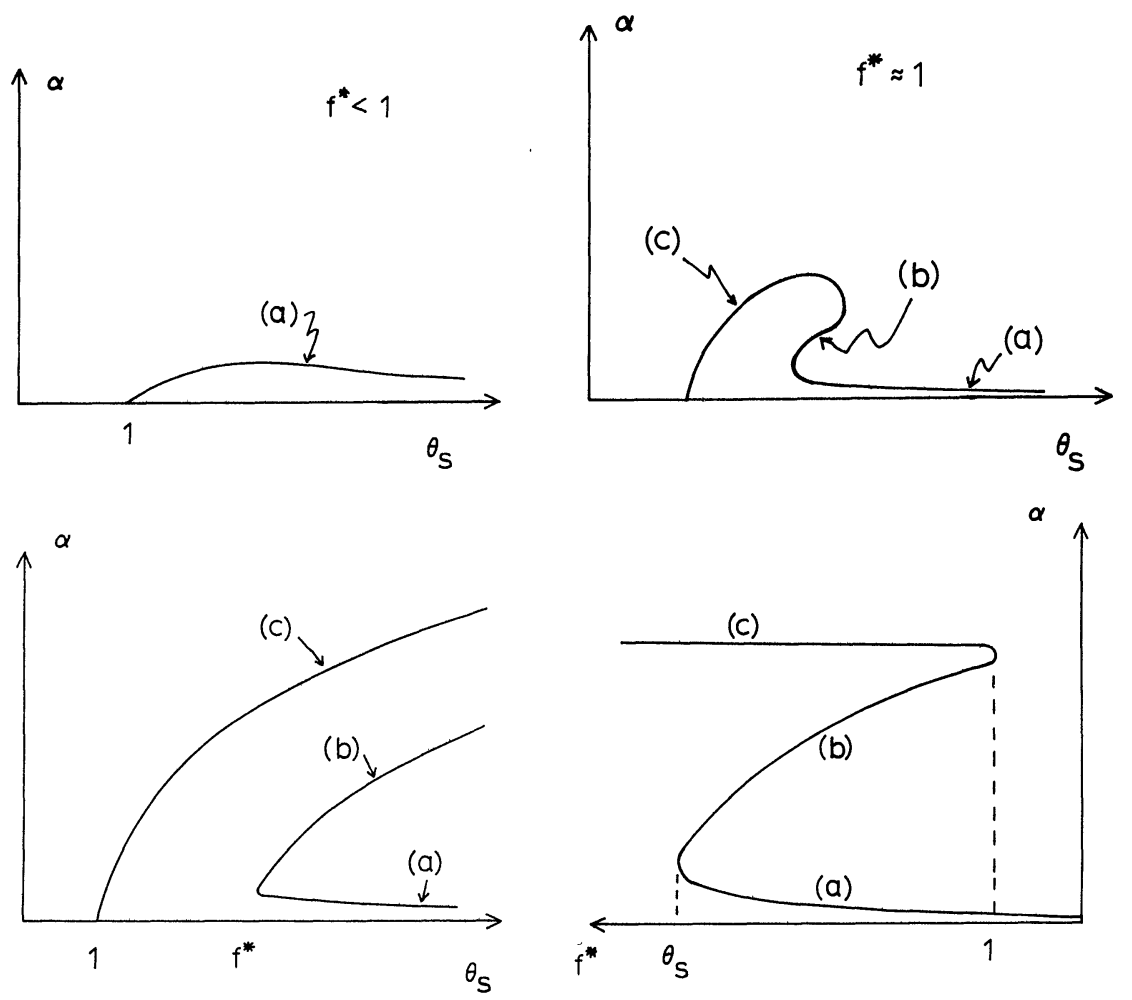

FIG. 4. (a)-(d): Bifurcation diagrams for the heaving coefficient $\alpha$ versus surface cooling $\theta_{s}$ for the case of fine soils (a), intermediate soils (b), and coarse soils (c); (d) $\alpha$ versus $f^{*}$ at fixed $\theta_{s}>1$. 


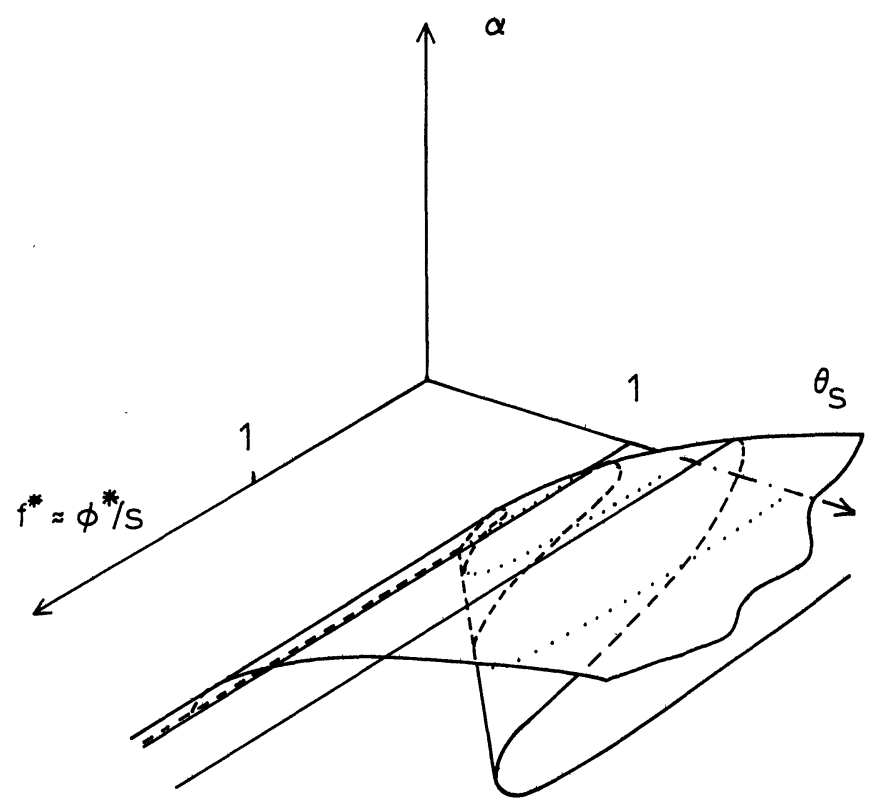

FIG. 5. A three-dimensional view of heave parameter $\alpha$ versus surface cooling $\theta_{s}$, and texture parameter $f^{*} \equiv \varphi^{*} / s$ (see $\left.\S 4\right)$.

$f$ and $1-\chi$ decrease. Thus lens formation will occur in the pressure boundary layer, and this happens at $z^{*}$, where using (3.14) and (3.16),

$$
-\left(f_{l}-1\right) \exp \left[-\Lambda\left(z_{l}-z^{*}\right)\right] \approx-\left[\psi_{l} f_{l}-1\right],
$$

where we have written $\psi_{l}=1-\chi_{l}=1-\chi\left(W_{l}\right)$, that is,

$$
z_{l}-z^{*} \approx \frac{1}{\Lambda} \ln \left[\frac{f_{l}-1}{\psi_{l} f_{l}-1}\right]
$$

Since (cf. (3.34)) $f_{l}>1$ for $\alpha>0$, we require $\psi_{l} f_{l}>1$ for successive lens formation to occur, and this happens "instantaneously." In practice, the interval between lens formations will presumably be controlled by the freezing relaxation time between lenses, $t \sim O\left(1 / \Lambda^{2}\right)$. With $[U] \sim 10^{-6} \mathrm{~cm} \mathrm{~s}^{-1},[z] \sim 10 \mathrm{~cm},[t] \sim 10^{7} \mathrm{~s}$, then $[t] / \Lambda^{2} \sim 10^{3} \mathrm{~s}$ if $\Lambda \sim 10^{2}$, consistent with Fig. 4 of [7]. The lens spacing is then $O(1 / \Lambda) \sim 10^{-2}$, or dimensionally $1 \mathrm{~mm}$.

Lensing is suppressed if $f_{l} \approx 1$, which is case (c) ( $\left.b \gg 1\right)$, corresponding to coarse soils (since $g^{*}$ is low). In this case, we should suppose that step freezing with a frozen fringe can only possibly lead to a surface ice layer. But with a superimposed load, even this first lens would require satisfaction of (3.6), and it seems reasonable to suppose that secondary heaving simply does not occur in this case. Heaving is also suppressed if $\psi_{l} \ll 1$, i.e., $W_{l} \approx n$, but this again is typically case (c) since if $W_{l}\left(=g_{l}\right) \approx n$, then $g_{l}>g^{*}$.

4. Discussion. Our main result is shown in Fig. 5. To try and understand it, let us recollect some important parameters. The main one is $\beta$, proportional to the saturated hydraulic conductivity, and varying from $\sim 10^{6}$ for gravel to $\sim 1$ for silts or clays. The critical water fraction $g^{*}=n /(\beta \gamma)^{1 / \gamma}$ plays an important role in the analysis: with $n=0.4, g^{*}$ varies from about 0.08 for gravel (with $\gamma=10$ ) to about 0.32 for clay. The parameter $f^{*}$ is actually $f\left(g^{*}\right)$, where $f$ is the dimensionless characteristic function. 
Now the dimensional version of this function is $P_{0} f(W)$, that, however, contains an imposed dependence on $P_{0}$. To include the overburden as an explicit parameter, we write

$$
s=P_{0} / \sigma, \quad P_{0} f(W)=\sigma \varphi(W),
$$

where $\sigma$ is a stress scale; thus $f=\varphi / s$, and $\varphi$ is a dimensionless function, dependent only on soil type. A simple assumption is that $\varphi$ is independent of soil type, which is manifested only through the stress scale $\sigma$. Then $\sigma$ is low for gravel, high for silt and clay. The parameter $f^{*}$ is thus actually $f\left(g^{*}\right)=\varphi\left(g^{*}\right) / s$. It is convenient to represent Fig. 5 in terms of the independent parameters $\varphi^{*}=\varphi\left(g^{*}\right)$ representing soil type, and $s$, representing loading. The result is shown in Fig. 6 , which is drawn by sketching Fig. $4(\mathrm{~d})$ at various values of $s$. The critical "knees" of the curve occur at $f^{*}=\theta_{s}$ and $f^{*}=1$, that is, $\varphi^{*}=\varphi_{c}^{*}$ and $\varphi^{*}=s$, where $\varphi_{c}^{*}$ is given by

$$
\varphi_{c}^{*}=s \theta_{s}=\left(\rho L / T_{0}\right)(\Delta T / \sigma),
$$

using (2.25) and (2.36), and $\Delta T$ is the surface cooling. Evidently, $\varphi_{c}^{*}$ is independent of the applied load, but depends (like $\varphi^{*}$ ) on soil type. Then, for fixed undercooling $\Delta T$, the condition $\varphi^{*}<\varphi_{c}^{*}$, which defines the range of existence of solutions of type (a), is independent of $s$, which explains the position of the second knee of the curve. At the intersection of $\varphi^{*}=\varphi_{c}^{*}=s, \alpha \rightarrow 0$, and heaving is suppressed since then $\theta_{s}<1$. For larger $\Delta T, \varphi_{c}^{*}$ is increased and the range of heavable loads is increased.

The existence of multiple solutions is reminiscent of similar situations in, for example, thermal runaway. It is a natural hypothesis that case (b) is unstable and hence unrealisable, so that there is a hysteresis loop between (a) and (c). Case (c), however, does not lead to ice lens formation and thus is not associated with heaving.

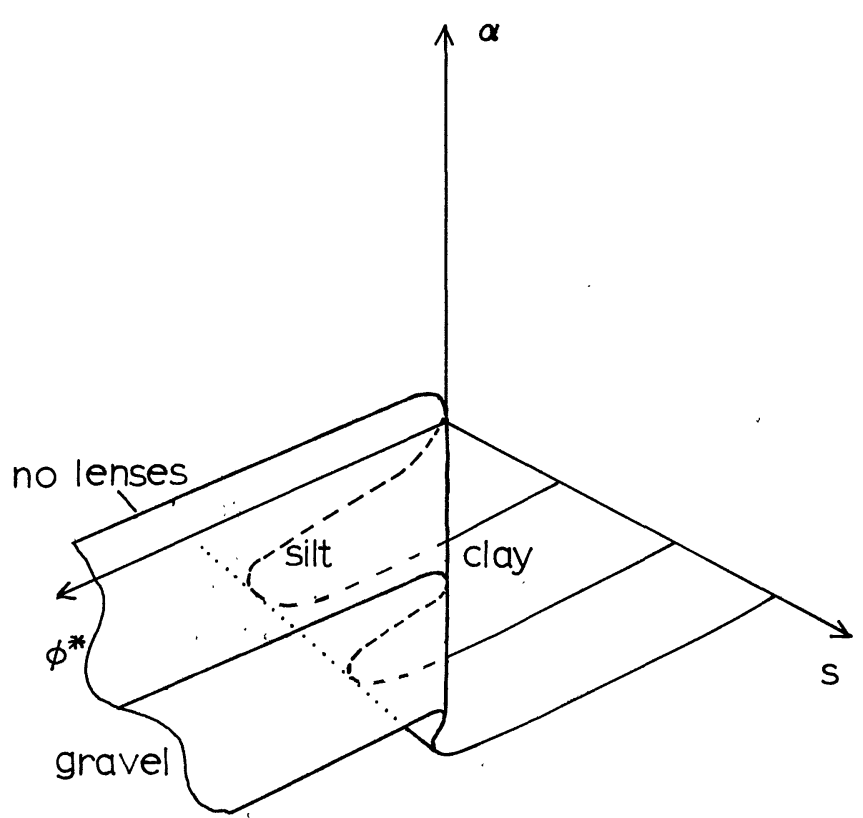

FIG. 6. A regime map based on Fig. 4 for different soils, measuring heave rate parameter $(\alpha)$ versus soild texture parameter $\left(\varphi^{*}\right)$ and load parameter $(s)$. The figure indicates no heaving for large $\varphi^{*}$ (gravel), large heaving for moderate loads for intermediate $\varphi^{*}$ (silt), and small heave rates for large loads (clay). In addition, for a fixed soil, heave is effectively suppressed at a critical value of the load parameter $\left(s=\varphi_{c}^{*}\right)$. 
In that case, only the case (a) branch, indicated by solid lines in Fig. 6, has any relevance. If this is the case, then we can interpret Fig. 6 as follows. First, we expect $\varphi^{*}$ to be high for low $g^{*}$, i.e., for gravel, and low for clays. Also, $\sigma$ is low for gravel, high for clay, so that at fixed $P_{0}, s$ is high for gravel, low for clay. Consequently, for small $\varphi^{*}$ (clay), $\alpha$ is very small, so that the rate of heave is small. On the other hand, heaving occurs for $s<\varphi_{c}^{*}$, that is $P_{0}<\varphi_{c}^{*} \sigma$. Since $\sigma$ is high for clay, large loads can be heaved. As $\varphi^{*}$ increases, there is a comparatively sudden jump near $\varphi^{*}=\varphi_{c}^{*}$, where $\alpha$ increases sharply. Thus silts can heave rapidly, but (since $\sigma$ is lower) only lower loads. For $\varphi^{*}>\varphi_{c}^{*}$ (gravel), heaving does not occur. These observations basically reproduce statement (a) of $\S 3$.

Apart from these overall observations, our analytic results mimic features of O'Neill and Miller's numerical results. As pointed out after (3.19), case (a) can be fully analysed even when similarity is not invoked. Then $v_{i} \ll 1$, and satisfaction of (3.17) with (3.18) and (3.3) 1,2 yields a linear profile for $f$, with

$$
\begin{aligned}
& z_{l}-z_{f} \approx\left(z_{f}-z_{b}\right) \theta_{s} / \theta_{b}-H, \\
& f_{l} \approx \theta_{s}-H \theta_{b} /\left(z_{f}-z_{b}\right) .
\end{aligned}
$$

The resultant linear temperature profile was assumed by Gilpin [2], and also found by O'Neill and Miller [7]. The pressure and temperature profiles found here correspond to observation (c) of $\S 3$. For clays, the initial similarity solution rapidly relaxes to a quasisteady state given by (4.3) and (4.4), where for $H \ll 1, v_{i}$ given by (3.16) is essentially constant (observation (d) in $\S 3$ ). The sensitivity to the load is manifested through the dependence of $v_{i}$ through $B(3.16)$, on $W_{l}(3.12)$, i.e., on $f_{l}$, which is determined by $\theta_{s}$ (4.4) which is proportional to $P_{0}^{-1}(2.25)$. We have already mentioned successive lens formation (following (3.35)).

The regime map, Fig. 5 or 6 , suggests that as the load is increased for a given soil, heaving will be rapidly suppressed at a critical value of $P_{0}=\sigma \varphi^{*}$. For a given soil, for which the soil-water characteristic function and the hydraulic conductivity $k_{h}$ are known, this critical value is in principle measurable, and could provide one means of testing the current theory.

5. Conclusions. Analysis of the O'Neill-Miller model is possible on the basis of the "large activation exponent" assumption that $\gamma \gg 1$, i.e., there is strong variation of permeability. This analysis yields completely explicit approximate results that are in qualitative agreement with O'Neill and Miller's [7] computed results. They also seem to be consistent with some basic observed characteristics of heaving soils. A feature of the results is the coexistence at some values of overburden and subcooling of three possible freezing modes, two of which involve lensing, the other of which does not. It is not at present known whether both lensing solutions are physically realisable, although it is plausible that the solution of case (b) is not.

The existence of explicit results of the type presented here suggests ways of parametrising laboratory and numerical experiments by means of regime diagrams of the type shown in Figs. 5 and 6. Another is indicated in Fig. 7 that might serve (for a particular soil) as a regime map: it is a projection of Fig. 5, using the definitions of $f^{*} / s$ and $\theta_{s}$. Further consideration of such maps is deferred to future work, together with consideration of such features as soil compressibility and solutes. It is interesting to note that introduction of $n=n\left(p_{e}\right)$ into (2.26) leads to cross derivatives in a diffusion-type equation for $(W, p)^{T}$. However, it is easy to check that the diffusion matrix has positive eigenvalues when $n^{\prime}\left(p_{e}\right)<0$, as is realistic. 


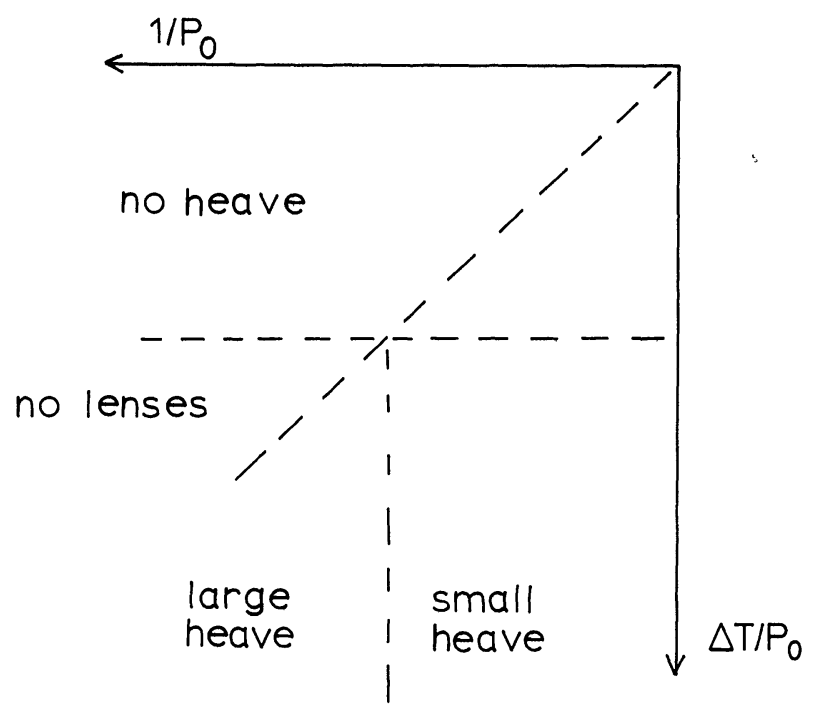

FIG. 7. A projection of the regime map, Fig. 4, on to the plane $\alpha=0$. For a given soil, $\theta_{s} \propto \Delta T / P_{0}$, $\varphi^{*} / s \propto 1 / P_{0}$, where $\Delta T$ is the surface cooling. This suggests ways of testing the current theory for particular soils.

Acknowledgments. I thank Rex Westbrook for discussions concerning the material in this paper.

\section{REFERENCES}

[1] D. A. Drew, Mathematical modelling of two-phase flow, Ann. Rev. Fluid Mech., 15 (1983), pp. 261-291.

[2] R. R. GiLPin, A model for the prediction of ice lensing and frost heave in soils, Water Resour. Res., 16 (1980), pp. 918-930.

[3] D. Hillel, Fundamentals of Soil Physics, Academic Press, New York, 1980.

[4] K. A. Jackson, D. R. Uhlmann, And B. Chalmers, Frost heave in soils, J. Appl. Phys., 37 (1966), pp. 848-852.

[5] R. D. Miller, Freezing phenomena in soils, in Applications of Soil Physics, D. Hillel, ed., Academic Press, New York, 1980, pp. 254-299.

[6] K. O'NeILL, The physics of mathematical frost heave models: a review, Cold Reg. Sci. Tech., 6 (1983), pp. 275-291.

[7] K. O'Neill AND R. D. Miller, Exploration of a rigid ice model of frost heave, Water Resour. Res., 21 (1985), pp. 281-296.

[8] P. J. Williams AND T. P. BURT, Measurement of hydraulic conductivity of frozen soils, Canad. Geotech. J., 11 (1974), pp. 647-650.

[9] M. G. WORSTER, Solidification of an alloy from a cooled boundary, J. Fluid Mech., 167 (1986), pp. 481-501. 